\title{
Icaritin, an inhibitor of beta-site amyloid cleaving enzyme-1, inhibits secretion of amyloid precursor protein in APP-PS1- HEK293 cells by impeding the amyloidogenic pathway
}

\author{
Fei Feng ${ }^{\text {Equal first author, 1 }}$, Yuanyuan Li ${ }^{\text {Equal first author, 2 }}$, Nanqu Huang ${ }^{2}$, Yong Luo ${ }^{\text {Corresp. } 1}$ \\ ${ }^{1}$ Department of Neurology, The First People's Hospital of Zunyi \& Third Affiliated Hospital of Zunyi Medical University, Zunyi, Guizhou, China \\ 2 National Drug Clinical Trial Institution, The First People's Hospital of Zunyi \& Third Affiliated Hospital of Zunyi Medical University, Zunyi, Guizhou, China \\ Corresponding Author: Yong Luo \\ Email address: luoyongtt@163.com
}

\section{Background}

Icaritin (ICT) is a prenylflavonoid derivative from Epimedium brevicornum Maxim. ICT has been shown to have neuroprotective effects. We investigate how ICT affects secretion of amyloid precursor protein (APP).

\section{Methods}

We exposed APP-PS1-HEK293 cells to ICT to investigate its effect on beta-site amyloid cleaving enzyme (BACE)1. Cell viability was evaluated by MTT and lactate dehydrogenase (LDH) assays. The half-maximal inhibitory concentration $\left(\mathrm{IC}_{50}\right.$ ) of ICT for BACE1 was measured using fluorescence resonance energy transfer. Effects of ICT on the mRNA expression of APP were assessed by quantitative polymerase chain reaction, and protein expression was measured by western blotting and immunofluorescence.

\section{Results}

ICT inhibited BACE1 activity and $\mathrm{IC}_{50}$ was $5.70 \pm 1.09 \mu \mathrm{M}$. Compared with the control group, at ICT concentrations of $5 \mu \mathrm{M}$ and $10 \mu \mathrm{M}$, the viability increased and LDH leakage decreased in APP-PS1-293 cells. Also, mRNA expression of A disintegrin and metalloproteinase domain-containing protein 10 (ADAM10) increased, while that of BACE1 and presenilin-1 (PS1) decreased, upon ICT treatment. Western blotting and immunofluorescence confirmed that protein expression of ADAM10, BACE1, and PS1 showed the same trend. Expression of the APP fragments SAPP $\beta$ and C-terminal fragment $\beta$ decreased, while that of SAPP $\alpha$ increased, upon ICT treatment. Expression of amyloid $\beta$ peptides in APP-PS1-HEK293 cells was lower in ICT-treated groups compared with that in the control group.

\section{Conclusions}

Icaritin, as a BACE1 inhibitor, inhibits APP secretion in APP-PS1-HEK293 cells by impeding the amyloidogenic pathway. 


\section{Original Research}

2

3 Icaritin, an inhibitor of beta-site amyloid cleaving enzyme-1, inhibits secretion of amyloid

4 precursor protein in APP-PS1-HEK293 cells by impeding the amyloidogenic pathway

5

6 Fei Feng 1 , Yuanyuan $\mathrm{Li}^{2}$, Nanqu Huang ${ }^{2}$, Yong Luo ${ }^{1}$

$8{ }^{1}$ Department of Neurology, The First People's Hospital of Zunyi \& Third Affiliated Hospital of

9 Zunyi Medical University, Zunyi, Guizhou, China

10 2National Drug Clinical Trial Institution, The First People's Hospital of Zunyi \& Third Affiliated

11 Hospital of Zunyi Medical University, Zunyi, Guizhou, China

Corresponding author: Yong Luo, 98, North Phoenix Road, Zunyi, Guizhou, 563003, China; e- 
ABSTRACT

\section{Background}

Icaritin (ICT) is a prenylflavonoid derivative from Epimedium brevicornum Maxim. ICT has been shown to have neuroprotective effects. We investigate how ICT affects secretion of amyloid precursor protein (APP).

\section{Methods}

We exposed APP-PS1-HEK293 cells to ICT to investigate its effect on beta-site amyloid cleaving enzyme (BACE)1. Cell viability was evaluated by MTT and lactate dehydrogenase (LDH) assays. The halfmaximal inhibitory concentration $\left(\mathrm{IC}_{50}\right)$ of ICT for BACE1 was measured using fluorescence resonance energy transfer. Effects of ICT on the mRNA expression of APP were assessed by quantitative polymerase chain reaction, and protein expression was measured by western blotting and immunofluorescence.

\section{Results}

ICT inhibited BACE1 activity and $\mathrm{IC}_{50}$ was $5.70 \pm 1.09 \mu \mathrm{M}$. Compared with the control group, at ICT concentrations of $5 \mu \mathrm{M}$ and $10 \mu \mathrm{M}$, the viability increased and LDH leakage decreased in APP-PS1-293 cells. Also, mRNA expression of A disintegrin and metalloproteinase domain-containing protein 10 (ADAM10) increased, while that of BACE1 and presenilin-1 (PS1) decreased, upon ICT treatment. Western blotting and immunofluorescence confirmed that protein expression of ADAM10, BACE1, and PS1 showed the same trend. Expression of the APP fragments sAPP $\beta$ and C-terminal fragment $\beta$ decreased, while that of sAPP $\alpha$ increased, upon ICT treatment. Expression of amyloid $\beta$ peptides in APP-PS1-HEK293 cells was lower in ICT-treated groups compared with that in the control group.

\section{Conclusions}

Icaritin, as a BACE1 inhibitor, inhibits APP secretion in APP-PS1-HEK293 cells by impeding the amyloidogenic pathway.

Keywords Alzheimer's disease; icaritin; amyloid- $\beta$ peptides; beta-site amyloid cleaving enzyme 1; presenilin-1; amyloid precursor protein 


\section{INTRODUCTION}

Alzheimer's disease (AD) is a degenerative disease of the central nervous system characterized by progressive cognitive dysfunction and behavioral impairment. According to a report from Alzheimer's Disease International, there are $\sim 9.5$ million dementia patients in China currently and this number may exceed 16 million by 2030 (International 2016). Due to a lack of efficacious therapeutic drugs, AD will be a major public health problem in China in the future. The exact etiology of $\mathrm{AD}$ is incompletely understood. Amyloid- $\beta$ peptides (A $\beta$ Ps) are generated through sequential cleavage of amyloid precursor protein (APP) by beta-site amyloid cleaving enzyme (BACE)1 and $\gamma$-secretase [presenilin-1 (PS1)], which play pivotal roles in AD pathogenesis (Querfurth \& LaFerla 2010). However, a series of clinical trials on AßPs have failed, and research on AD has faltered (Voytyuk et al. 2018).

The Chinese pharmaceutical chemist and 2015 Nobel Prize Laureate Youyou Tu and her research team studied artemisinin extracted from the stems and leaves of Artemisia annua L. They found that artemisinin can be used to treat malaria. Therefore, finding treatment for intractable diseases such as AD using phytomedicine could be a viable strategy. Icaritin (ICT) is a prenylflavonoid derivative from Epimedium brevicornum Maxim. The molecular weight of ICT is 386.4 , it is soluble in fat, and can pass through the blood-brain barrier readily. ICT has been shown to enhance proliferation of mesenchymal stem cells (Lim et al. 2018), attenuate oxidative stress and the neuronal toxicity induced by A $\beta$ Ps (Wang et al. 2007; Wu et al. 2014). Furthermore, natural flavonoids can inhibit BACE-1 activity and reduce the level of secreted A $\beta$ Ps. Therefore, ICT could be an efficacious drug against AD.

Previously, we demonstrated that ICT can improve memory impairment in a rat model of AD by reducing A $\beta \mathrm{P}$ accumulation (Feng et al. 2017). To further explore the regulatory mechanism of ICT on AßP accumulation (Fig 1), we designed a study to probe how ICT modulates expression of BACE1 and $\gamma$ secretase, and its potential neuroprotective effects, in vitro.

\section{MATERIALS AND METHODS}

\section{Materials}


APP-PS1-HEK293 cells were purchased from Shanghai Enzyme-linked Biotechnology (Shanghai, China). Icaritin (purity $>98.3 \%$ ) was obtained from Nanjing Zelang Medical Technologies (Nanjing, China). 3-(4,5-dimethylthiazol-2-yl)-2,5-diphenyltetrazolium bromide (MTT) was purchased from Biosharp (Hefei, China). A lactate dehydrogenase (LDH) assay kit was obtained from Beyotime (Shanghai, China). An electrochemiluminescence (ECL) kit was purchased from Thermo Fisher (Waltham, MA, USA). A BACE1 Fluorescence Resonance Energy Transfer (FRET) Assay kit was obtained from Panvera (Madison, WI, USA). A PrimeScript ${ }^{\mathrm{TM}}$ RT Reagent kit and SYBR ${ }^{\mathrm{TM}}$ Premix Ex Taq were purchased from TaKaRa Biotechnology (Shiga, Japan). Antibodies against anti-BACE1, antipresenilin 1 , anti- $\mathrm{A} \beta \mathrm{P}_{1-40}$, anti-A $\beta \mathrm{P}_{1-42}$, anti-glyceraldehyde 3-phosphate dehydrogenase (GAPDH), and goat anti-rabbit immunoglobulin (Ig)G H\&L (DyLight 488) were obtained from Abcam (Cambridge, UK). Anti-C-terminal fragment $\beta$ (CTF $\beta$ ) antibody was purchased from Biolegend (San Diego, CA, USA). AntisAPP $\beta$ (6A1) antibody and anti-sAPPa (2B3) antibody were obtained from Immuno-Biological Laboratories (Fujioka-Shi, Japan). Horseradish peroxidase-conjugated goat anti-mouse or anti-rabbit IgG antibody was purchased from Abmart (Shanghai, China).

\section{BACE1 inhibition assay}

The BACE1 inhibition assay was executed using a BACE1 FRET kit according to manufacturer instructions. A stock solution of ICT in dimethyl sulfoxide was prepared (20 mmol/L). The sample was diluted further in assay buffer to prepare a $3 \times$ concentration of test compounds. $10 \mu \mathrm{L}$ of BACE1 substrate (a new "red" FRET peptide substrate based on the "Swedish" mutant, $3 \times$ concentration) was added to $10 \mu \mathrm{L}$ of $3 \times$ concentration of each test compound in separate wells of a black $96-w e l l$ microplate and mixed gently. Then, $10 \mu \mathrm{L}$ of $3 \times$ BACE1 was added to each well to start the reaction. Plates were incubated for $90 \mathrm{~min}$ at $25^{\circ} \mathrm{C}$ in the dark. Then, $10 \mu \mathrm{L}$ of sodium acetate was added to each well to stop the reaction. Finally, fluorescence measurements were undertaken with a multimode microplate reader (Multiskan ${ }^{\mathrm{TM}}$ GO; Thermo Scientific) at an excitation wavelength of $545 \mathrm{~nm}$ and emission wavelength of $585 \mathrm{~nm}$. The half-maximal inhibitory concentration $\left(\mathrm{IC}_{50}\right)$ was calculated by plotting the obtained relative fluorescence unit per hour (RFU/h) against the logarithmic of the inhibitor concentration. The measured 
inhibition data were analyzed in Prism 7 (GraphPad, La Jolla, CA, USA) by nonlinear regression (curve fitting).

\section{Cell culture}

APP-PS1-HEK293 cells subcultured by 1:3, generations were maintained in Dulbecco's modified Eagle's medium (DMEM)/high glucose (SH30022.01B; Hyclone, Logan, MT, USA) supplemented with $10 \%$ fetal bovine serum (04-001-1A; Hyclone) and penicillin-streptomycin (SV30010; Hyclone) at $37^{\circ} \mathrm{C}$ in a humidified atmosphere of $5 \% \mathrm{CO}_{2}$.

\section{Cell viability by the MTT and LDH assays}

Cell viability was determined by MTT and LDH assays. Briefly, APP-PS1-HEK293 cells cultured in 96-well plates (5000/well) were treated with different concentrations of ICT for $16 \mathrm{~h}$. The medium was removed and $50 \mu \mathrm{L}$ of MTT reagent $(0.5 \mathrm{mg} / \mathrm{mL})$ in DMEM was added to each well. Then, cells were incubated for $3 \mathrm{~h}$ at $37^{\circ} \mathrm{C}$. After dissolving formazan crystals with $150 \mu \mathrm{L}$ of dimethyl sulfoxide, absorbance at $570 \mathrm{~nm}$ was measured on a microplate reader (Multiskan GO). An LDH assay kit was used to evaluate injury/damage to cells according to manufacturer protocols, and absorbance at $490 \mathrm{~nm}$ was measured.

\section{Quantitative polymerase chain reaction (qPCR)}

Total RNA from cultured cell samples were extracted using TRIzol ${ }^{\mathrm{TM}}$ Reagent (Sigma-Aldrich, Saint Louis, MD, USA) through a series of rinse, elution, and centrifugation steps. RNA samples were reverse-transcribed using the PrimeScript RT Reagent kit (RR047A; TaKaRa Biotechnology). SYBR Premix Ex Taq (RR820A; TaKaRa Biotechnology) was used following manufacturer guidelines for measurement of mRNA expression of BACE1, PS1, and $\alpha$-Secretase. All primer sequences are summarized in Table 1. Fluorescence signals were amplified and detected using a StepOnePlus ${ }^{\mathrm{TM}}$ sequence detector (Thermo Scientific) and analyzed with Nanodrop 2000 (Thermo Scientific). The cycle threshold $(\mathrm{Ct})$ for each sample was averaged from triplicates. The $2^{-\Delta \Delta \mathrm{Ct}}$ approach was used whereby 
133

134

135

136

137

138

139

140

141

142

143

144

145

146

147

148

149

150

151

152

153

154

155

156

157

158

159

fluorescence signals were normalized to the corresponding housekeeping gene $G A P D H$.

\section{Western blotting}

APP-PS1-HEK293 cell lysates were prepared. Briefly, after 30 min of incubation with lysis buffer at $4^{\circ} \mathrm{C}$, insoluble material was removed by centrifugation at $12,000 \mathrm{rpm}$ for $30 \mathrm{~min}$ at $4^{\circ} \mathrm{C}$. The protein concentration of the lysate was determined using a bicinchoninic acid protein assay kit (GK5012; Generay, Shanghai, China). Equal amounts $(10 \mu \mathrm{g})$ of each sample were separated by sodium dodecyl sulfate-polyacrylamide gel electrophoresis (P0012AC; Beyotime, Shanghai, China) and transferred to polyvinylidene difluoride (PVDF) membranes (IPVH00010; Merck, Kenilworth, NJ, USA). PVDF membranes were blocked for $1 \mathrm{~h}$ in a 5\% $(w / v)$ fat-free milk solution in TBST (Tris buffer containing $0.5 \%$ $(v / v)$ Tween-20). PVDF membranes were incubated overnight at $4{ }^{\circ} \mathrm{C}$ in $5 \%(w / v)$ bovine serum albumin solution in TBST with antibodies (all purchased from Abcam) against BACE1 antibody (1:1000 dilution; ab2077), anti-PS1 (1:1000; ab76083), anti-A disintegrin and metalloproteinase domain-containing protein 10 (ADAM10; 1:1000; ab1997), anti-AßP ${ }_{1-40}$ (1:1000; ab20068), and anti-GAPDH (1:1000; ab8245). PVDF membranes were washed thrice (10-min each time) by TBST and incubated with horseradish peroxidase-conjugated goat anti-mouse or anti-rabbit IgG secondary antibodies (1:5000; Abmart, Berkeley Heights, NJ, USA). Then, PVDF membranes were washed with TBST thrice and visualized using the ECL kit. Band intensity was quantified with Quantity One (Bio-Rad Laboratories, Hercules, CA, USA).

\section{Immunofluorescence}

After treatment with different concentrations of ICT for $16 \mathrm{~h}$, cells plated on coverslips were fixed with methanol for $20 \mathrm{~min}$ at $4^{\circ} \mathrm{C}$. They were permeabilized with $0.1 \%$ Triton $\mathrm{X}-100$ in phosphatebuffered saline for $5 \mathrm{~min}$ at room temperature, and treated with blocking medium $(5 \%$ bovine serum albumin in phosphate-buffered saline) for $30 \mathrm{~min}$. Then, they were incubated with antibodies against antiBACE1 (1:1000 dilution), anti-PS1 (1:1000), and anti-ADAM10 (1:1000) at room temperature for $2 \mathrm{~h}$. Immune-reacted primary antibody was detected following 1-h incubation in the dark at room temperature 
160

161

162

163

164

165

166

167

168

169

170

171

172

173

174

175

176

177

178

179

180

181

182

183

184

185

186

with a secondary antibody conjugated with Dylight 488 (1:1000 dilution). Cells were further stained with 4',6-diamidino-2-phenylindole (C1002; Beyotime) for $2 \mathrm{~min}$ in the dark at room temperature and washed. Then, they were placed onto microscope slides in mounting medium. Observations were carried out using a fluorescence microscope (BX43F; Olympus, Tokyo, Japan).

\section{Statistical analyses}

Data are the mean $\pm \mathrm{SEM}$ from at least three independent experiments. Comparisons of more than two groups were made with one-way ANOVA, followed by Dunnett's post hoc test. SPSS v22.0 (IBM, Armonk, NY, USA) was employed for statistical analyses $P<0.05$ was considered significant.

\section{RESULTS}

\section{$\mathrm{IC}_{50}$ of ICT for BACE1}

ICT inhibited BACE1 activity and the $\mathrm{IC}_{50}$ was $5.70 \pm 1.09 \mu \mathrm{M}$ (Fig 2). ICT inhibition was like that seen for other flavonoids, such as quercetin and apigenin (Sathya et al. 2012).

\section{Protective effect of ICT in APP-PS1-293 cells}

Cell viability was measured by the MTT assay and cell-membrane damage was determined according to LDH leakage. These parameters were used to ascertain the neuroprotective effects of ICT in APP-PS1-293 cells. Compared with the control group, at ICT concentrations of $5 \mu \mathrm{M}$ and $10 \mu \mathrm{M}$, the viability increased and LDH leakage decreased in APP-PS1-293 cells. These results suggested a neuroprotective effect of ICT in APP-PS1-293 cells at concentrations of $5 \mu \mathrm{M}$ and $10 \mu \mathrm{M}$ (Fig. 3).

\section{Effect of ICT on the amyloidogenic pathway of APP in APP-PS1-HEK293 cells}

To investigate whether the amyloidogenic pathway is involved in the protective effects of ICT in APP-PS1-HEK293 cells, we detected some of the mRNA and proteins involved in this pathway.

First, we focused on changes in expression of $\alpha-, \beta$ - and $\gamma$-secretase. We noted a significant increase in expression of ADAM10 in the ICT group compared with that in the control group (Fig 4A, Fig 5, Fig 
6A). We observed a significant decrease in BACE1 expression in the ICT group compared with that in the control group (Fig 4B, Fig 5, Fig 6B). We documented a significant decrease in PS1 expression in the ICT group compared with that in the control group (Fig 4C, Fig 5, Fig 6C). These results suggested that ICT could promote the non-amyloidogenic pathway, and inhibit the amyloidogenic pathway, of APP.

To verify our results, we detected some APP fragments. Compared with the control group, protein expression of sAPP $\beta$ and CTF $\beta$ was reduced significantly, whereas that of sAPP $\alpha$ increased. Finally, we found that protein expression of $\mathrm{A} \mathrm{P}_{1-40}$ and $\mathrm{A} \beta \mathrm{P}_{1-42}$ in APP-PS1-HEK293 cells was lower in ICT-treated groups compared with that in the control group.

\section{DISCUSSION}

Epimedium is a genus of flowering plants in the family Berberidaceae. The main monomer component extracted from it, icariin (ICA), has been studied extensively. Zhang et al. used APPV717I transgenic mice to study the effect and mechanism of action of ICA on A $\beta$ P production. They found that ICA (30 and $100 \mu \mathrm{mol} / \mathrm{kg}$ per day) could reduce A $\beta$ P levels by decreasing expression of APP and BACE1 (Zhang et al. 2014). Li et al. found similar regulatory mechanisms in a study of ICA on cognitive impairments induced by permanent occlusion of bilateral common carotid arteries (PO-BCCAs) in rats. ICA reduced the level of A $\beta$ Ps in the rat hippocampus subjected to PO-BCCAs by reducing expression of BACE1 and increasing expression of ADAM10 ( $\mathrm{Li}$ et al. 2015). ICA also has a similar effect in tripletransgenic mice that are more able to mimic $\mathrm{AD}$. Chen et al. used $3 \times$ transgenic-AD mice to investigate the neuroprotective properties of ICA. They found that ICA reduced deposition of A $\beta$ P plaques in AD mice, and inhibited BACE1 expression.

The data stated above suggest that an extract of Epimedium had an inhibitory effect on BACE1 expression. Also, as a derivative of ICA, ICT, has a lower molecular weight and can penetrate the blood brain barrier readily (see Supplementary Material). Therefore, ICT has better drug-development capabilities than ICA. Previously, we demonstrated that ICT can improve memory impairment in a rat model of AD by reducing A $\mathrm{P}$ accumulation (Feng et al. 2017).

In the present study, we used swAPP695 and PSEN1dE9 double gene-transfected HEK293 cells. 
214

215

216

217

218

219

220

221

222

223

224

225

226

227

228

229

230

231

232

233

234

235

236

237

238

239

240

APP-PS1-HEK293 cells show high expression of APP and PS1 in SwAPP695 and PSEN1dE9, and can increase expression of A $\beta$ Ps significantly. Hence, APP-PS1-HEK293 cells can be used as an AD model.

First, we used a BACE1 FRET kit to determine the $\mathrm{IC}_{50}$ of ICT for BACE1. ICT inhibited the activity of BACE1 and the $\mathrm{IC}_{50}$ of ICT was $5.70 \pm 1.09 \mu \mathrm{M}$, similar to that of other flavonoids such as quercetin and apigenin (Sathya et al. 2012). Next, MTT and LDH assays were employed to detect the neuroprotective effects of ICT in APP-PS1-293 cells. We found that ICT had neuroprotective effects at 5 $\mu \mathrm{M}$ and $10 \mu \mathrm{M}$ in APP-PS1-293 cells. Then, we detected the effect of ICT on the amyloidogenic pathway of APP in APP-PS1-HEK293 cells. APP has two pathways in transmembrane secretion: nonamyloidogenic and amyloidogenic. The non-amyloidogenic pathway mainly produces soluble sAPP $\alpha$ by $\alpha$-secretase (ADAM10 and ADAM17) (Gupta \& Goyal 2016). Simultaneously, the CTF $\alpha$ of APP remains, and $\mathrm{CTF} \alpha$ can be cleaved further by $\gamma$-secretase (PS1) to produce APP intracellular domain CTF $\gamma$ (AICD) and p3 peptide (which does not aggregate). This metabolic pathway predominates in healthy status, and is required to maintain normal physiologic functions. Compared with the control group, the level of ADAM10 and SAPP $\alpha$ in the ICT group increased, suggesting that ICT promotes the non-amyloidogenic pathway. A $\beta$ Ps are produced via the amyloidogenic pathway. First, APP is secreted by BACE1 from the $\mathrm{N}$-termini of A $\beta$ Ps to produce a soluble APP N-terminal fragment ( $\mathrm{APP} \beta$ ) and a CTF $\beta$. Then, cleavage is continued by $\gamma$-secretase, resulting in a series of A PPs of varying lengths (39-43 amino acids) and AICD. When the balance between $\mathrm{A} \beta \mathrm{P}$ production (amyloidogenic pathway) and A $\beta \mathrm{P}$ clearance (nonamyloidogenic pathway) is broken off, it causes abnormal deposition of A $\beta$ Ps in the brain (Castro et al. 2019; Tiwari et al. 2019). In the present study, compared with the control group, expression of sAPP $\beta$, BACE1, PS1, and CTF $\beta$ in the ICT group decreased, indicating that ICT inhibits the amyloidogenic pathway.

The major findings of our study were that ICT could reduce production of $A \beta \mathrm{P}_{1-40}$ and $\mathrm{A} \beta \mathrm{P}_{1-42}$ through inhibition of expression of BACE1 and PS1 (which are the two most important enzymes in the amyloid metabolic pathway) and that expression of ADAM10 increased simultaneously (which is another cause of reduction of $\mathrm{A} \beta \mathrm{P}$ levels). $\mathrm{BACE} 1$ and $\gamma$-secretase have been considered to be potential targets for new therapeutic agents that block AßP generation (Coimbra et al. 2018; Kumar et al. 2018), though 
241

242

243

244

245

246

247

248

249

250

251

252

253

254

255

256

257

258

259

260

261

262

263

264

265

266

267

several issues must be addressed urgently (Voytyuk et al. 2018). Here, we showed that ICT can decrease $\mathrm{A} \beta \mathrm{P}$ levels and could be neuroprotective by reducing $\mathrm{A} \beta \mathrm{P}$ generation. Our previous in vivo study revealed that ICT can alleviate memory impairment in an AD model in rats (Feng et al. 2017) Taken together, ICT might be developed for AD therapy.

\section{CONCLUSIONS}

Our results indicated that ICT, as a BACE1 inhibitor, inhibits APP secretion in APP-PS1-HEK293 cells by impeding the amyloidogenic pathway.

\section{REFERENCES}

Castro MA, Hadziselimovic A, and Sanders CR. 2019. The vexing complexity of the amyloidogenic pathway. Protein Sci 28:1177-1193. 10.1002/pro.3606

Coimbra JRM, Marques DFF, Baptista SJ, Pereira CMF, Moreira PI, Dinis TCP, Santos AE, and Salvador JAR. 2018. Highlights in BACE1 inhibitors for Alzheimer's disease treatment. Front Chem 6:178. 10.3389/fchem.2018.00178

Feng F, Li Y, Tong H, Tu L, and Luo Y. 2017. Icaritin prevents neurotoxicity induced by amyloid- $\beta$ through increasing AMPK phosphorylation. Latin Am J Pharm 36:855-859.

Gupta A, and Goyal R. 2016. Amyloid beta plaque: a culprit for neurodegeneration. Acta Neurologica Belgica 116:445-450. 10.1007/s13760-016-0639-9

International AsD. 2016. Alzheimer's Disease International. World Alzheimer report 2016. Available at https://www.alz.co.uk/research/world-report-2016 (accessed 2016/09/20 2016/09/20).

Kumar D, Ganeshpurkar A, Kumar D, Modi G, Gupta SK, and Singh SK. 2018. Secretase inhibitors for the treatment of Alzheimer's disease: Long road ahead. Eur J Med Chem 148:436-452. 10.1016/j.ejmech.2018.02.035

Li WX, Deng YY, Li F, Liu B, Liu HY, Shi JS, and Gong QH. 2015. Icariin, a major constituent of flavonoids from Epimedium brevicornum, protects against cognitive deficits induced by chronic brain hypoperfusion via its anti-amyloidogenic effect in rats. Pharmacol Biochem Behav 138:40- 
48. 10.1016/j.pbb.2015.09.001

Lim RZL, Li L, Yong EL, and Chew N. 2018. STAT-3 regulation of CXCR4 is necessary for the prenylflavonoid icaritin to enhance mesenchymal stem cell proliferation, migration and osteogenic differentiation. Biochim Biophys Acta 1862:1680-1692. 10.1016/j.bbagen.2018.04.016

Querfurth HW, and LaFerla FM. 2010. Alzheimer's disease. N Engl J Med 362:329-344. 10.1056/NEJMra0909142

Sathya M, Premkumar P, Karthick C, Moorthi P, Jayachandran KS, and Anusuyadevi M. 2012. BACE1 in Alzheimer's disease. Clin Chim Acta 414:171-178. https://doi.org/10.1016/j.cca.2012.08.013

Tiwari S, Atluri V, Kaushik A, Yndart A, and Nair M. 2019. Alzheimer's disease: pathogenesis, diagnostics, and therapeutics. Int J Nanomed 14:5541-5554. 10.2147/ijn.S200490

Voytyuk I, De Strooper B, and Chavez-Gutierrez L. 2018. Modulation of gamma- and beta-secretases as early prevention against Alzheimer's disease. Biol Psychiatry 83:320-327. 10.1016/j.biopsych.2017.08.001

Wang Z, Zhang X, Wang H, Qi L, and Lou Y. 2007. Neuroprotective effects of icaritin against beta amyloid-induced neurotoxicity in primary cultured rat neuronal cells via estrogen-dependent pathway. Neuroscience 145:911-922. 10.1016/j.neuroscience.2006.12.059

Wu J, Xu H, Wong PF, Xia S, Xu J, and Dong J. 2014. Icaritin attenuates cigarette smoke-mediated oxidative stress in human lung epithelial cells via activation of PI3K-AKT and Nrf2 signaling. Food Chem Toxicol 64:307-313. 10.1016/j.fct.2013.12.006

Zhang L, Shen C, Chu J, Zhang R, Li Y, and Li L. 2014. Icariin decreases the expression of APP and BACE-1 and reduces the beta-amyloid burden in an APP transgenic mouse model of Alzheimer's disease. Int J Biol Sci 10:181-191. 10.7150/ijbs.6232 
Figure 1

Role of ICT in the metabolic pathway of amyloid precursor proteins (schematic).

(A) The plant is Epimedium brevicornum Maxim; (B) The chemical structure of ICT; (C) Role of ICT in the metabolic pathway of amyloid precursor proteins (schematic).

A

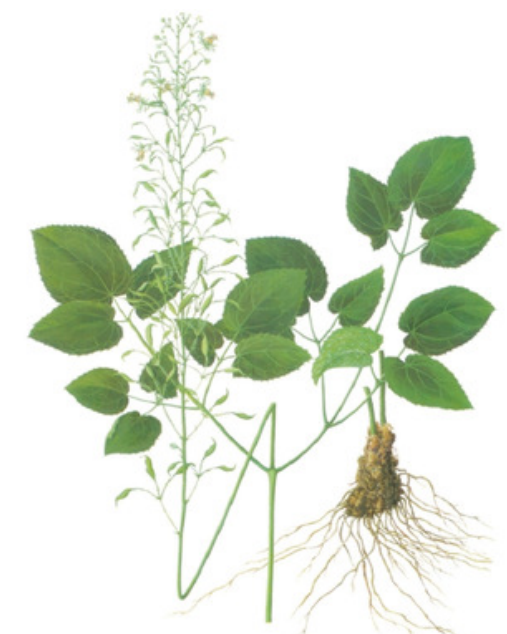

Epimedium brevicornum Maxim.
$\mathrm{B}$<smiles>COc1ccc(-c2oc3c(CC=C(C)C)c(O)cc(O)c3c(=O)c2O)cc1</smiles>

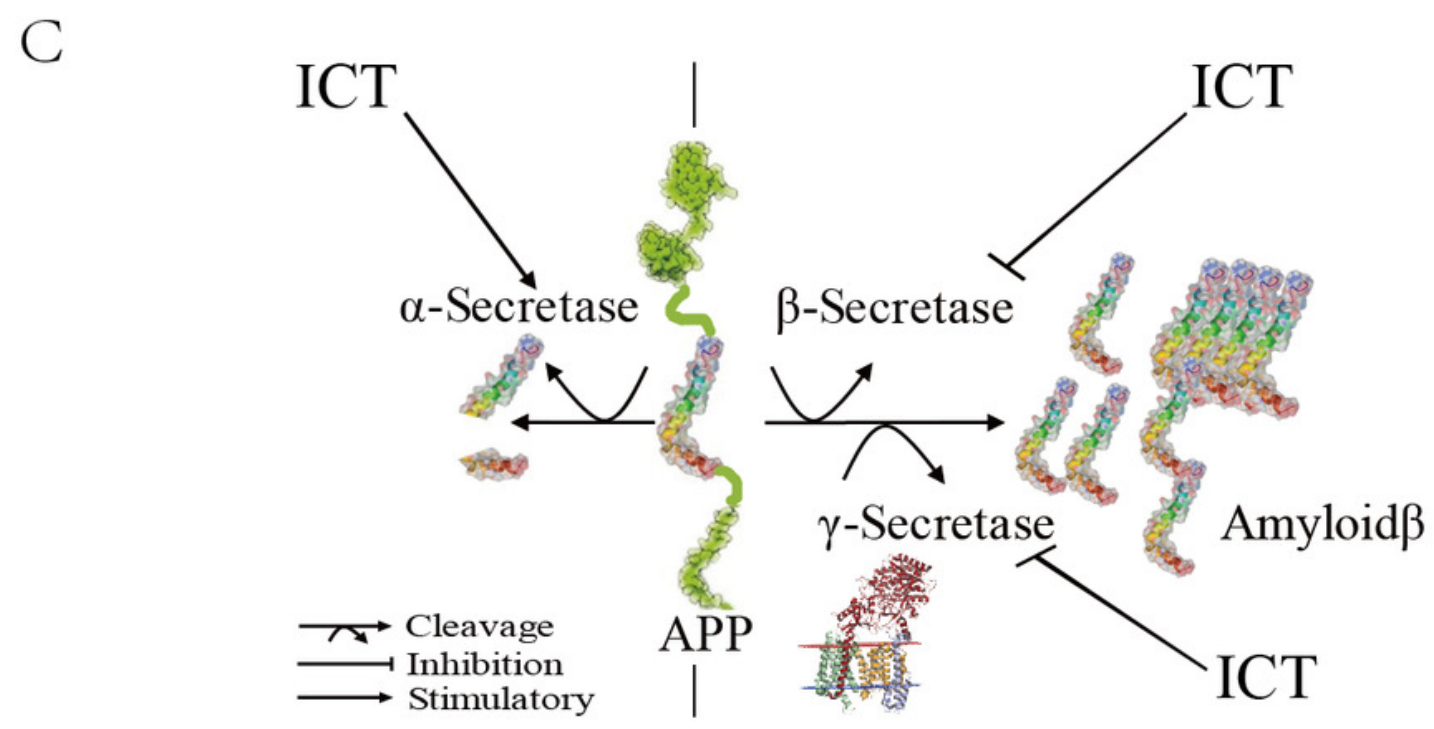


Figure 2

$I_{50}$ of ICT for BACE1. The BACE1 inhibition assay was executed using a BACE1 FRET kit.

$\mathrm{IC}_{50}$ values were calculated by plotting the obtained relative fluorescence unit per hour (RFU/h) against the logarithmic of the inhibitor concentration. Mean $\pm S E M, n=3$.

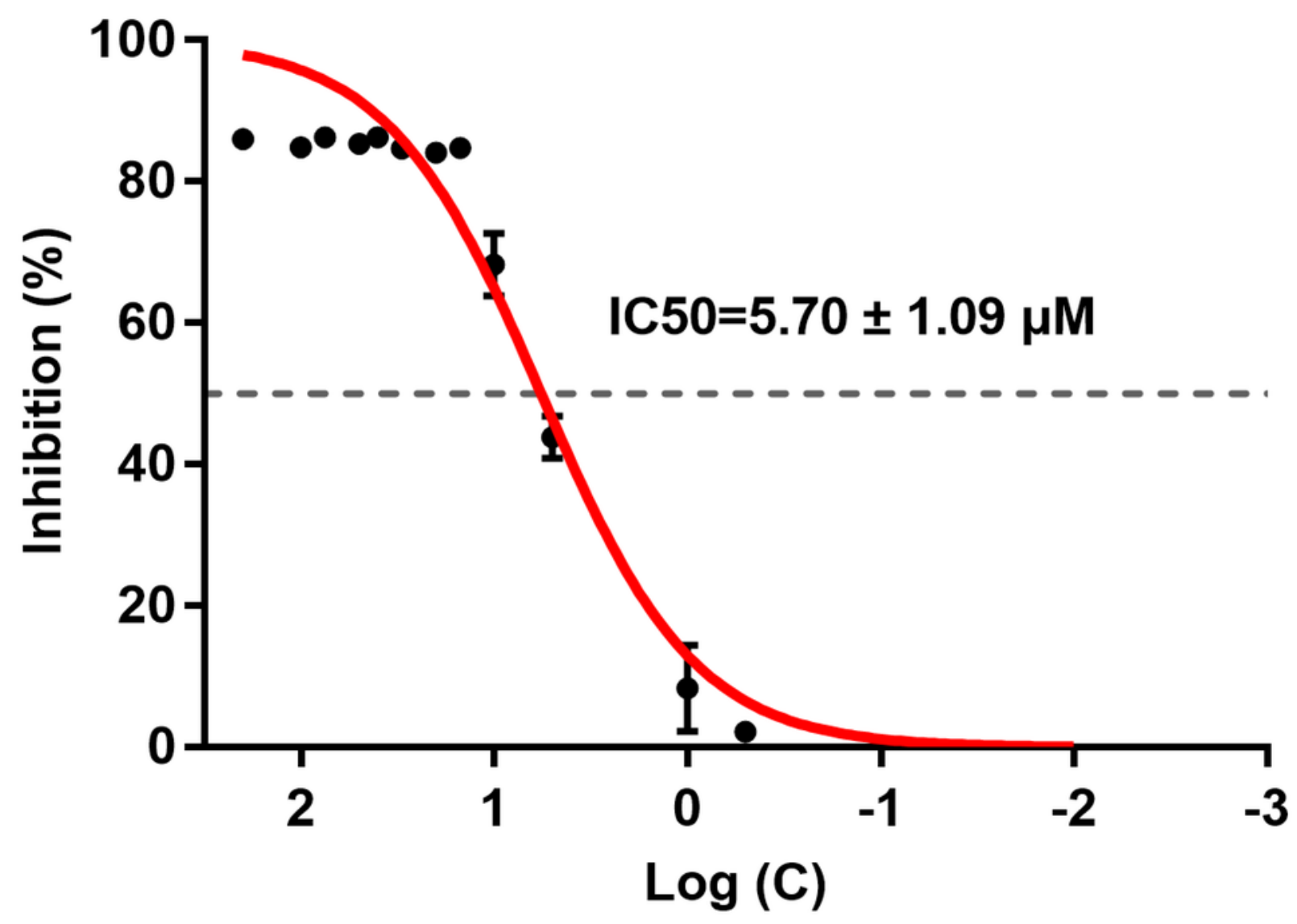


Figure 3

MTT and LDH assays in APP-PS1-293 cells.

Effects of ICT $(0.1,0.5,1,5,10,20,50,100 \mu \mathrm{M})$ on (A) cell viability by the MTT assay and (B)

LDH leakage ( ${ }^{*} P<0.05$ vs. control, mean \pm SEM, $\left.n=3\right)$.

A

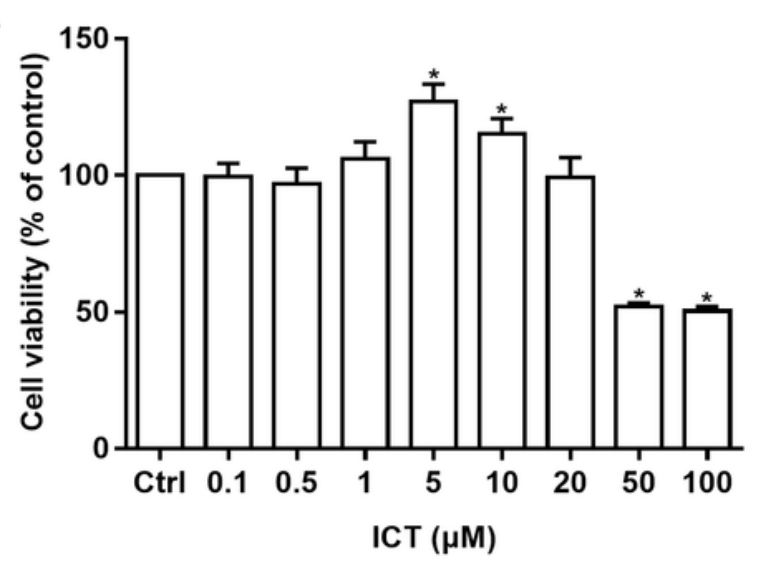

B

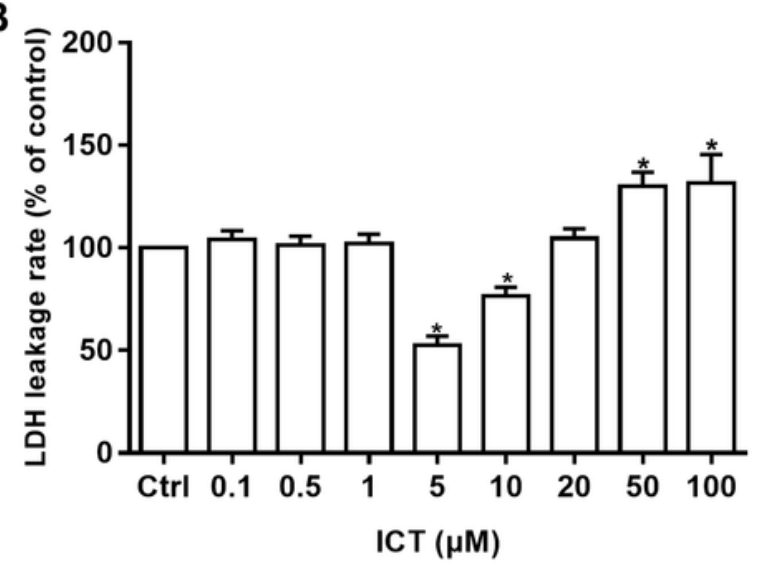


Figure 4

Effect of ICT $(0.5,5,10 \mu \mathrm{M})$ on mRNA expression of ADAM10, BACE1, and PS1 in APPPS1-HEK293 cells.

(A) ADAM10 mRNA; (B) BACE1 mRNA; (C) PS1 mRNA. mRNA expression of targets was normalized to that of GAPDH ( ${ }^{*} P<0.05$ vs. control, mean $\left.\pm \mathrm{SEM}, \mathrm{n}=3\right)$.
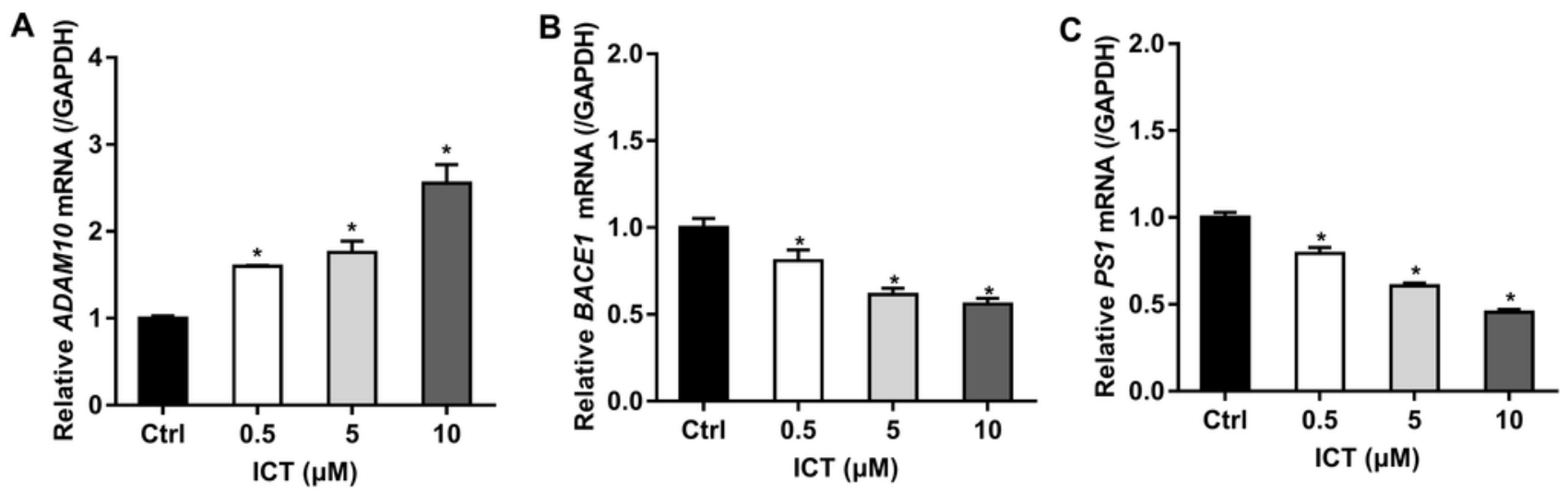
Figure 5

Effects of ICT on protein expression of the APP metabolic pathway in APP-PS1-HEK293 cells.

(A) Representative bands of SAPP $\alpha$, SAPP $\beta$, ADAM10, BACE1, PS1, ABP $P_{1-42}, A \beta P_{1-40}, C T F \beta$, and GAPDH; (B) relative protein expression of SAPP $\alpha$, SAPP $\beta, A D A M 10, B A C E 1, P S 1, A \beta P_{1-42}, A \beta P$ ${ }_{1-40}, \mathrm{CTF} \beta$, and GAPDH. The relative absorbance was normalized to that of GAPDH. ${ }^{*} P<0.05$ vs. control, mean \pm SEM, $n=3$. 
A

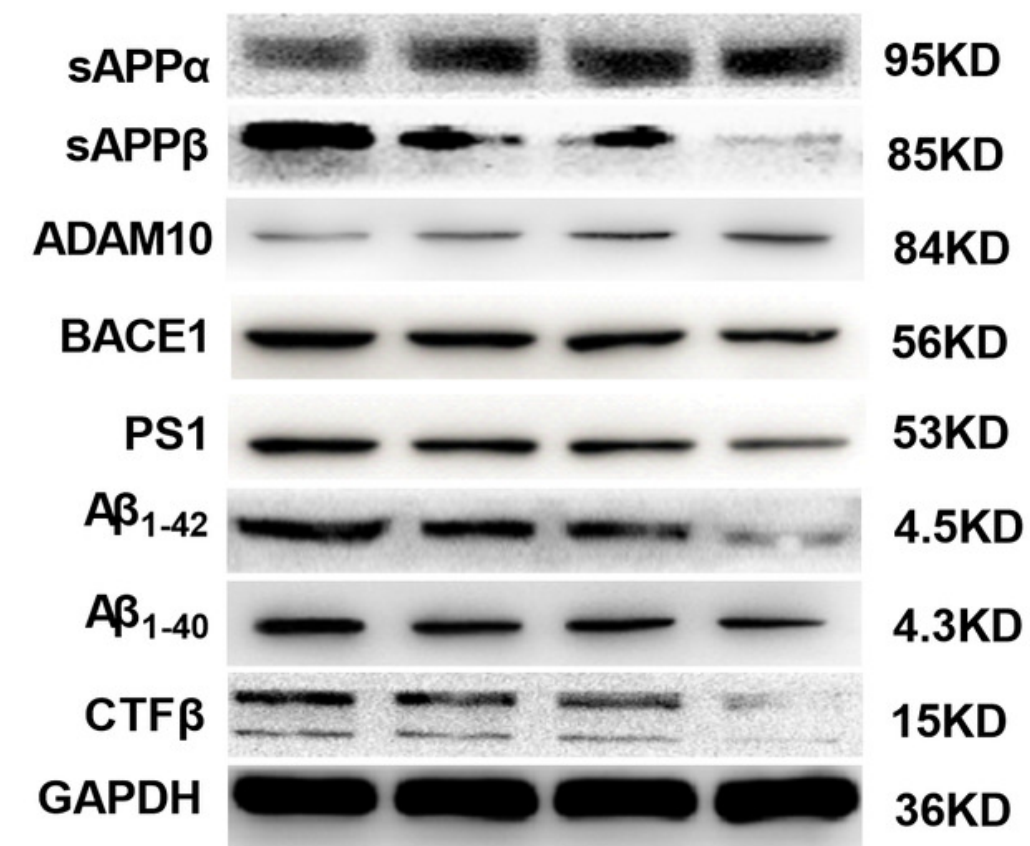

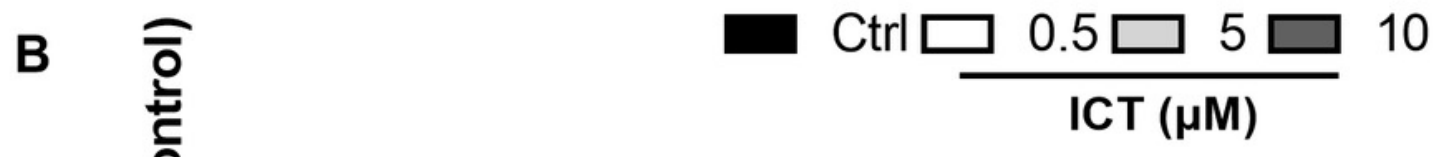




\section{Figure 6}

ICT reduced expression of BACE1 and PS1 and increased expression of ADAM10 in APPPS1-HEK293 cells.

Blue fluorescence represents the nuclei of APP-PS1-HEK293 cells, green fluorescence represents ADAM10, BACE1, and PS1, respectively. (A) ADAM10, (B) BACE1, (C) PS1. Scale bar is $20 \mu \mathrm{m}, \mathrm{n}=3$.
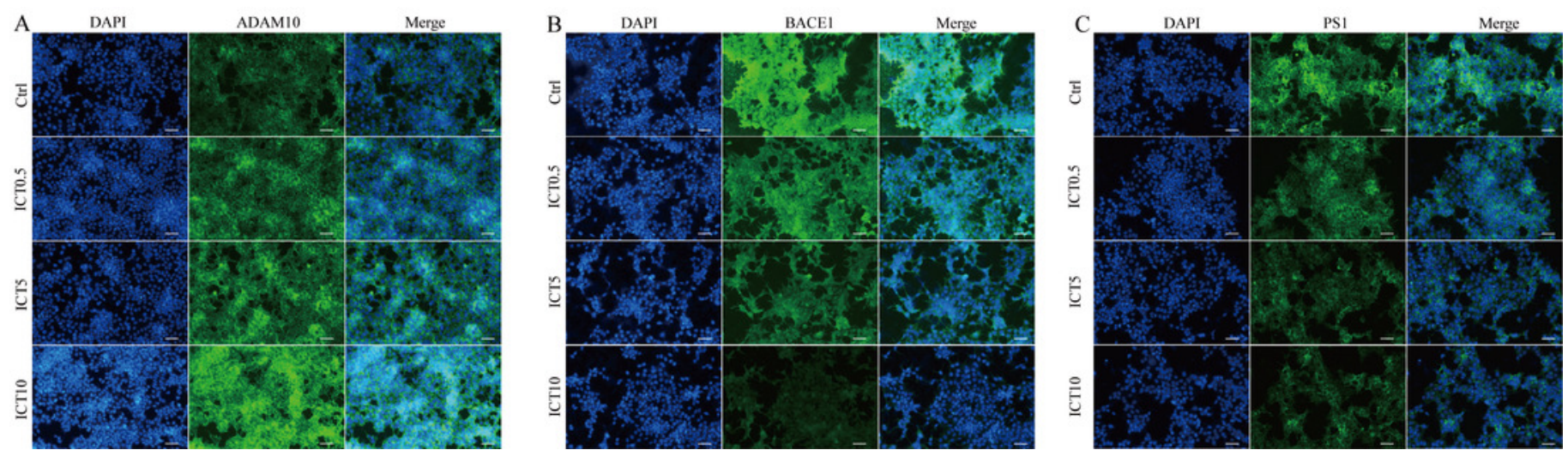


\section{Table 1 (on next page)}

Table. 1 The primer sequences used in the study. 
1 Table. 1 The primer sequences used in the study.

\begin{tabular}{|c|c|}
\hline Primer & Sequence $\left(5^{\prime}-3^{\prime}\right)$ \\
\hline BACE1 forward & GCGGGAGTGGTATTATGAAGTG \\
\hline BACE1 reverse & CCACGATGCTCTTGTCATAGTT \\
\hline PS1 forward & GAGCCCTGCACTCAATTCT \\
\hline PS1 reverse & CCAGGCATGGATGACCTTATAG \\
\hline$\alpha$-Secretase forward & CAGGAAGCTCTGGAGGAATATG \\
\hline$\alpha$-Secretase reverse & GAGACTTTGGGAGGTACATGAG \\
\hline GAPDH forward & GATGCTGGTGCTGAGTATGT \\
\hline GAPDH reverse & GCGGAGATGATGACCCTTT \\
\hline
\end{tabular}

